\title{
The neutrophil-to-lymphocyte ratio determines clinical efficacy of corticosteroids therapy in patients with COVID-19
}

Jingjing Cai

Wuhan University

Haomiao Li

Wuhan University

Ye-Mao Liu

Wuhan University

Changjiang Zhang

Wuhan University

Fang Lei

Wuhan University

Juan-Juan Qin

Wuhan University

Feng Zhou

Central South University

Xiaohui Song

Wuhan University

Liangjie Bai

Central South University

Jianghua Zhou

Wuhan University

Yan-Ci Zhao

Wuhan University

Lihua Zhu

Wuhan University

Peng Zhang

Central South University

Xin Zhang

Central South University

Juan Yang

Wuhan University

Yan-Xiao Ji 
Central South University

Guang-Nian Zhao

Central South University

\section{Zhigang Lu}

The First People's Hospital of Jingmen affiliated to Hubei Minzu University

\section{Liming Liu}

Ezhou Central Hospital

\section{Weiming Mao}

Huanggang Central Hospital

\section{Xiaofeng Liao}

Affiliated Hospital of Hubei University of Arts and Science

\section{Haofeng Lu}

The First Affiliated Hospital of Changjiang University

\section{Daihong Wang}

Xianning Central Hospital

\section{Xigang Xia}

Jingzhou Central Hospital

\section{Xiaodong Huang}

Wuhan Third Hospital \& Tongren Hospital of Wuhan University

\section{Xiang Wei}

Huazhong University of Science and Technology

\section{Jiahong Xia}

Huazhong University of Science and Technology

\section{Bing-Hong Zhang}

Renmin Hospital of Wuhan University

\section{Yufeng Yuan}

Zhongnan Hospital of Wuhan University

\section{Yibin Wang}

University of California

\section{Xiao-Jing Zhang}

Wuhan University

\section{Zhi-Gang She}

Wuhan University

Hongliang Li ( $\square$ lihl@whu.edu.cn )

Wuhan University

\section{Research Article}

Keywords: COVID-19, corticosteroids, inflammatory status, neutrophil-to-lymphocyte ratio, mortality 
Posted Date: August 4th, 2020

DOl: https://doi.org/10.21203/rs.3.rs-41841/v1

License: (c) (i) This work is licensed under a Creative Commons Attribution 4.0 International License. Read Full License

Version of Record: A version of this preprint was published at Cell Metabolism on February 1st, 2021. See the published version at https://doi.org/10.1016/j.cmet.2021.01.002. 


\section{Abstract}

Preliminary results from the RECOVERY trial indicated that dexamethasone usage markedly reduced death rate in COVID-19 patients receiving invasive mechanical ventilation. However, the overall reduction for the entire patient cohort in that trial was much more modest, indicating highly variable effects of corticosteroid usage among COVID-19 patients. While steroid treatment is known to have both clinical efficacy and detrimental adverse-effects, defining a clinic parameter that could guide the beneficial corticosteroid usage for treating COVID-19 remains an elusive, urgent, and critical unmet need in COVID19 therapy. Here, we undertook a multicentered retrospective study on a cohort of 12,862 confirmed COVID-19 cases from 21 hospitals in Hubei Province, China, including 3,254 received corticosteroid treatment and 9,608 received usual care without corticosteroid. We uncovered that the clinical benefits of corticosteroid use were closely associated with the neutrophil-to-lymphocyte ratio (NLR) measured at admission. Among participants with NLR $>6.12$ at admission, corticosteroid treatment was significantly associated with a lower risk of 60-day all-cause mortality of COVID-19 based on both Cox model with time-varying exposure and Marginal Structural Model. However, in patients with NLR $\leq 6.12$ at admission, corticosteroid treatment was no longer associated with reduced risk of all-cause death, but rather with increased risks of severe adverse effects, particularly in hyperglycemia and infection. In diabetic patients with COVID-19, corticosteroid treatment was associated with increased glycemia, but not with a higher risk of 60-day mortality. Therefore, our study has uncovered NLR as a clinical indicator to stratify COVID19 patients in their response to corticosteroid therapy. This finding may assist clinical evaluation and future randomized controlled trials to establish proper guidelines for corticosteroid therapy in COVID-19 patients.

\section{Introduction}

Corticosteroids have been used for over half a century to treat overwhelming inflammatory diseases and lung injury, but whether steroid use is clinically efficacious is still a matter of intensive debate1-3. For COVID-19, particularly the severe cases, the therapeutic efficacy of corticosteroids remains inconclusive and highly controversial4,5. The international guidelines regarding the use of corticosteroids for COVID19 was inconsistent, mainly based on the inconclusive clinical outcomes of corticosteroid use during the previous outbreaks of SARS and MERS6,7, as well as its known risk of prolonging viremia due to impaired immune system to clear invading pathogens 8,9. However, widespread usage of corticosteroids continues in patients with COVID-19 to treat overwhelming inflammation and cytokine-related pneumonia progression, especially in those suffering from life- threatening acute respiratory distress syndrome (ARDS) and septic shock10.

Recently, the RECOVERY trial led by the University of Oxford published its preliminary results that dexamethasone, a corticosteroid, may reduce one-third of COVID-19-associated death in patients on invasive ventilators 11,12. After that, WHO stated to update the clinical guide for dexamethasone use in 
patients with severe or critical disease under close clinical supervision. However, when evaluated from the entire cohort, the effect of dexamethasone in death reduction was much more moderate, indicating clearly that the clinical efficacy of corticosteroid treatment for COVID-19 is highly variable. Although ventilator usage is a sign of critical stage for COVID-19, it cannot be used as an effective method for patient stratification for corticosteroid therapy as it can be influenced by many factors including ventilator accessibility 13 . Therefore, it is critical to identify a specific and robust clinical indicator to assist physicians in determining whether and which patients infected with this novel coronavirus could benefit from corticosteroid therapy.

Prominent changes in the proportion of blood cell subsets, particularly in immune cells, have been recognized as the hallmark for systemic inflammation response and also are closely associated with COVID-19 outcomes14-16. Given that the primary benefit from corticosteroid use on pneumonia is suppressing the overwhelming inflammatory response, changes in circulating immune cells may be

important for guiding corticosteroid application in patients with COVID-19. Besides, routinely blood cell test is one of the most common clinical tests available for all hospitalized patients with very low cost and efficiency. To expand the applicability of the indicator in regions with different medical conditions, we sought to derive a powerful indicator from blood cell test. Here, we conducted a large retrospective study to analyze the associations of corticosteroid use with mortality and to identify clinical indicators from blood cell tests which could predict the therapeutic efficacy of corticosteroids in 12,862 patients hospitalized due to COVID-19.

\section{Results}

\section{Baseline characteristics of participants}

The study cohort consisted of COVID-19 patients admitted between December 30th, 2019 and April 17th, 2020, to 21 hospitals in Hubei, China that were designated to treat COVID-19 patients. There were 12,862 patients with COVID-19 who met the eligibility criteria for analysis. Among the study population, 3,254 (25.3\%) received corticosteroid therapy (corticosteroid group) and 9,608 (74.7\%) without corticosteroid therapy (non-corticosteroid group). The clinical characteristics of patients enrolled in the study were shown in Table 1. Participants receiving corticosteroid were older (61 [IQR, 49-69] years versus 57 [IQR, $45-67$ ] years, $P<0.001)$ and had a higher number of males $(56.2 \%$ versus $45.8 \%)$ than those in the noncorticosteroid group (Table 1). There was a higher prevalence of pre- existing hypertension $(37.2 \%$ versus $32.0 \%, P<0.001)$, diabetes mellitus $(18.3 \%$ versus $15.3 \%, P<0.001)$, chronic obstructive pulmonary disease (COPD; $1.6 \%$ versus $1.0 \%, P=0.005)$ and heart failure $(0.9 \%$ versus $0.4 \%, P=0.004)$ in the corticosteroid group than those without corticosteroid therapy (Table 1). The frequencies of neutrophil and leukocyte count increase, lymphocyte count decrease, C- reactive protein (CRP) increase, procalcitonin increase, creatine kinase (CK) increase, creatinine increase, and alanine transaminase (ALT) increase were significantly higher in the corticosteroid group than the non-corticosteroid group (Table 1). 
Accumulated evidence from the clinical trial suggested favorable immune response modulation by lowto-moderate dose corticosteroids could be beneficial to patients with critical conditions17-19. However, the critical condition wasn't exactly identified. To identify an indicator associated with disease severity and also can determine the therapeutic efficacy of corticosteroids in patients with COVID-19, we first identified blood cell parameter has the strongest association with 60-day in-hospital mortality in patients with COVID-19; After blood cell parameter was identified, we selected three candidate threshold values for the parameter with different capabilities to predict clinical outcome in patients with COVID-19; Finally, we further defined an appropriate severity threshold values for initiating corticosteroids therapeutic in patients with COVID-19.

In the first step, to determine indicators significantly associated with 60-day in-hospital mortality, ten parameters from complete blood cells were included in a multivariate logistic regression analysis. Using a multivariate logistic regression model on the dataset of 12,862 patients with COVID-19, the lymphocyte count decrease $(\mathrm{OR}, 5.17 ; 95 \% \mathrm{Cl}, 4.33-6.17 ; P<0.001)$ and neutrophil count increase $(\mathrm{OR}, 5.00 ; 95 \% \mathrm{Cl}$, 3.96-6.29; $P<0.001$ ) were the top two factors significantly and positively associated with mortality (Table 2). Remarkedly, using LASSO regression, a regularization method creates parsimonious model, the circulating immune cells were key determinants associated with the mortality. Among immune cells, neutrophil and lymphocyte counts were also identified as the top two factors (Table 2). These findings were consistent with previous studies20,21 and indicated that systemic immune response was among the most critical factors related to the clinical outcomes in this cohort of patients with COVID-19.

To further estimate whether the levels of immune cells were also associated the use of corticosteroid in clinic, we conducted the same analyses on the entire cohort. Intriguingly, we found that neutrophil increase and lymphocyte decrease were also the top two covariates significantly associated with use of corticosteroid during hospitalization.

Considering neutrophil and lymphocyte counts indicated immune status and their significant association with clinical outcomes, we adapted an existing index neutrophil to lymphocyte ratio (NLR), a well-known index for patient's inflammatory status and risk of death22-24, and to further explore its potential as an indicator guided the use of corticosteroid. The $\mathrm{C}$-statistic analysis showed continuous NLR has a high prognostic significance for death, with the area under of receiver-operating characteristic curve [AUROC] of 0.87 (95\% Cl, 0.86-0.88) in the dataset with 12,862 patients with COVID-19 (Table 3).

\section{NLR value as an indicator of clinical outcome in COVID-19}

Since the NLR values exhibited a robust prognostic effect in our study population, we further investigated the candidate NLR threshold values in C-statistic analysis regarding their specificity and sensitivity in predicting mortality in all patients. When the model sensitivity (above 0.9) was considered over specificity, a threshold value of NLR at 3.13 demonstrated a high sensitivity (0.91), but a low specificity (0.62) and accuracy $(0.64[95 \% \mathrm{Cl}, 0.63-0.65])$ in predicting mortality in our cohort of COVID-19 patients; In contrast, when the model specificity (above 0.9 ) were considered over sensitivity, a threshold value of NLR at 8.31 had the high specificity (0.91) ) and accuracy $(0.89[95 \% \mathrm{Cl}, 0.89-0.90])$, but inadequate sensitivity (0.64). 
Finally, when specific and sensitivity were both considered, a threshold of NLR at 6.12 was identified with the highest Youden index while both good sensitivity, specificity, and accuracy were achieved (sensitivity, 0.75; specificity, 0.85; accuracy, 0.85) (Table 3).

Positive predictive values (PPV) and negative predictive values (NPV) for each of these three NLR threshold values indicated an imbalanced dataset in terms of predictive accuracy. Thus, balanced accuracies were calculated. The results showed a similar pattern that a threshold value of NLR 6.12 had the highest balanced accuracy for predicting clinical mortality in patients with COVID-19 (Table 3).

\section{Primary outcomes based on NLR-dependent patient stratifications}

To evaluate the efficiency of each NLR cutoff value in differentiating clinical benefits of corticosteroid therapy, we test the association between corticosteroid therapy and clinical outcomes across the different sub-cohorts in our patient population stratified based on NLR threshold values of 3.13, 6.12 and 8.31 as described earlier. The clinical characteristics of patients in the sub-cohorts stratified based on NLR values were listed in Table 4. Consistent with the observations made in the complete cohort, the patients treated with corticosteroid were more severe and had higher percentages of comorbidities than the patients not received corticosteroid treatment in each NLR based sub-cohorts.

We applied two models, i.e., the Cox time-varying model and the marginal structural model (MSM), to account for immortal time bias and time-varying confounders by indications plus immortal time bias, respectively, to estimate the association setween corticosteroid use and 60-day all-cause death in each NLR based sub-cohorts. The imbalanced confounders between the corticosteroid and the noncorticosteroid groups were adjusted.

When the study cohort was stratified using a cut-off value of NLR 3.13 into two sub-cohorts with NLR $\leq$ 3.13 and NLR $>3.13$, the Cox time-varying model (adjusted HR, $0.62 ; 95 \% \mathrm{Cl}, 0.49-0.77 ; P<0.001$ ) showed a significantly lower risk of COVID-19 death in the corticosteroid group than the non- corticosteroid group in the sub-cohort of patients with NLR > 3.13 (Table 5). However, this association was not supported by the MSM model. In contrast, there were no significant associations between corticosteroid therapy with the risk of 60-day all-cause death in the sub-cohort of patients with an NLR $\leq 3.13$ using either Cox timevarying or MSM model (Table 5). Therefore, NLR 3.13 were not a suitable threshold for differentiating clinical benefits of corticosteroid therapy in patients with COVID-19.

When the study cohort was stratified using an NLR cut-off value of $\mathbf{8 . 3 1}$, in the sub-cohort with NLR > 8.31 corticosteroid treatment was associated with a significantly lower incidence and risk of 60-day allcause death compared to the non-corticosteroid users by both Cox time-varying model (adjusted HR, 0.55 ; $95 \% \mathrm{Cl}, 0.42-0.70 ; P<0.001$ ) and MSM (adjusted HR, 0.52; 95\% $\mathrm{Cl}, 0.38-0.70 ; P<0.001$ ). In the sub-cohort with NLR $\leq 8.31$, corticosteroid treatment was not significantly associated with the risk of COVID-19 death using either the Cox time-varying model or MSM model (Table 5). These results suggested that COVID-19 patients with NLR >8.31showed significant benefit from corticosteroid treatment. 
Finally, when patients were divided into two subgroups by an NLR at 6.12, a cutoff with the highest balanced accuracy, after adjusted for baseline and time-varying confounders, both Cox time- varying model (adjusted HR, 0.58; 95\% Cl, 0.46-0.73; $P<0.001$ ) and MSM analysis (adjusted OR, $0.56 ; 95 \% \mathrm{Cl}, 0.42-$ $0.73 ; P<0.001)$ indicated that the in-hospital use of corticosteroid was associated with a lower risk of $60-$ day all-cause death compared to corticosteroid nonuse (Table 5). Once the NLR was lower than 6.12, there was no significant association between corticosteroid use with the risk of all-cause death (Table 5). Thus, the NLR at 6.12 not only showed optimal sensitivity and specificity to predict the risk of mortality, but also can stratify patients with the different therapeutic outcome from corticosteroid therapy.

These results demonstrated that the clinical benefits and therapeutic outcome of corticosteroid therapy for COVID-19 were significantly influenced by the patient's immune status. Importantly, given the highest balanced accuracy for predicting mortality risk, the consistency observed from both models, and, in particular the remarkable differences in the opposite clinical outcome observed in the stratified subcohorts, a cut-off value of NLR at 6.12 appeared to be the optimal level for patient stratification for survival benefits from corticosteroid therapy.

\section{Validating NLR cutoff at 6.12 in subgroup and sensitivity analyses}

To further validate the performance of NLR based sub-cohort stratification using 6.12 cut-off value for the clinical benefits of corticosteroid treatment, we performed subgroup and sensitivity analyses using the following patient cohorts: (1) patients on mechanical ventilation with versus without corticosteroid therapy; (2) patients receiving a most commonly applied corticosteroid, methylprednisolone (methylprednisolone group), versus those not taking any forms of corticosteroids (nonmethylprednisolone group); and (3) patients taking corticosteroids versus those not taking corticosteroids after patients from two randomly selected hospitals were removed. Both Cox time- varying and MSM models were conducted on those subgroups for sensitivity analyses.

We first analyzed the associations between corticosteroid use and the risk of death among the patients who received mechanical ventilation during hospitalization. From both Cox time-varying model and MSM analysis, corticosteroid therapy was consistently associated with a significantly reduced risk of 60-day mortality among patients with an NLR $>6.12$ at admission with adjusted $\mathrm{HR}$ of $0.53(95 \% \mathrm{Cl}, 0.36-0.78 ; P$ $=0.001)$ in the Cox time-varying model and adjusted OR of $0.59(95 \% \mathrm{Cl}, 0.37-0.94 ; P<0.001)$ (Table 6). Notably, among patients with NLR $\leq 6.12$, corticosteroid therapy was not associated with any significant change in risk of 60-day all-cause death compared to no- corticosteroid treatment (Table 6).

Methylprednisolone is the most commonly prescribed corticosteroids in the study cohort. We further conducted subgroup analysis to compare the incidences and risks of 60-day all-cause death between patients taking methylprednisolone (methylprednisolone group) and those not taking any forms of corticosteroids (non-methylprednisolone group). Consistent with the earlier analysis using the entire cohort, for patients with NLR above 6.12, methylprednisolone therapy was associated with a significantly lower risk of 60-day all-cause death compared to the patients in the non- methylprednisolone group based on the Cox time-varying model (adjusted HR, 0.52; $95 \% \mathrm{Cl}, 0.39-0.68 ; P<0.001$ ) and MSM (adjusted OR, 
0.46; 95\% Cl, 0.31-0.69; $P<0.001$ ) (Table 6). Again, no significant associations between

methylprednisolone use and 60-day all-cause death risk were observed among patients with NLR $\leq 6.12$ (Table 6).

In a sensitivity analysis by randomly removing two hospital sites, for patients with NLR $>6.12$, corticosteroid treatment demonstrated a lower risk of 60-day all-cause death than no corticosteroid therapy from both the Cox time-varying model and MSM (Table 6). No significant associations between corticosteroid use and mortality risk were found in the patients with NLR $\leq 6.1$ (Table 6). Thus, all these findings in subgroup and sensitivity analyses were consistent with our observations in the entire cohort, and further supported the accuracy and robustness of NLR cutoff at 6.12 to guide patient stratification for corticosteroid treatment.

\section{Corticosteroid use in patients stratified based on NLR cutoffs}

Among the 3,254 individuals who received corticosteroids, the major type of corticosteroids was methylprednisolone, which accounted for $97.1 \%$ of the corticosteroids treated patients, followed by prednisolone (10.7\%) and hydrocortisone (1.0\%) (Table 7). Methylprednisolone was also the most frequently administered corticosteroid in each subgroup of patients separated by different NLR cutoffs (Table 7).

The median duration of corticosteroids treatment was $8.0(95 \% \mathrm{Cl}, 5.0-15.0)$ days. Notably, the initiated time of corticosteroid therapy was as early as at $1.0(95 \% \mathrm{Cl}, 0.0-4.0)$ day after admission. The median level of the daily dosage was relatively low, at $40.0(95 \% \mathrm{Cl}, 31.1-40.0) \mathrm{mg}$ of methylprednisoloneequivalent dosage with the median accumulated dose at $320.0(95 \% \mathrm{Cl}, 180.0-580.0) \mathrm{mg}$ (Table 7). Intriguingly, the accumulated dosages of corticosteroid in patients with an NLR above each cutoff were gradually increased along with the elevation of NLR values (Table 7). It is apparent that patients with an NLR above the cutoff had earlier initiating time, higher daily and accumulated doses, and longer corticosteroid treatment duration than those below the cutoffs (Table 7).

\section{Adverse effects of corticosteroid treatment in patients with an NLR $>6.12$}

In previous clinical trials, the adverse effects observed from corticosteroids therapy included hyperglycemia, gastroduodenal bleeding, hypernatremia, and infection19,25. Here, we analyzed the incidences and risks of those potential adverse effects related to corticosteroid treatment in the NLR stratified sub-cohorts. Among the subgroup with an NLR > 6.12 at admission, corticosteroid treatment was associated with higher incidences of hyperglycemia requiring treatment (IRR, 2.33 [1.95-2.78]; IR, 0.79 versus $0.34 ; P<0.001$ ), infection requiring acceleration of antibiotics (IRR, 2.51 [2.18-2.90]; IR, 1.29 versus $0.51 ; P<0.001)$ and fungal infection requiring antifungal treatment (IRR, 1.67 [1.21-2.31]; IR, 0.19 versus $0.11 ; P=0.002$ ) (Table 8 ). The higher risks of hyperglycemia requiring treatment and infection needing antibiotic acceleration in the corticosteroid treatment group were consistently observed in both the Cox time-varying model and the MSM (Table 8). Thus, despite a significantly lower risk of 60-day all- 
cause death in patients taking corticosteroid, the adverse effects of hyperglycemia and infection should be closely monitored in patients with NLR $>6.12$.

\section{Adverse effects of corticosteroid treatment in patients with an NLR $\leq 6.12$}

Among patients with an NLR $\leq 6.12$, the incidences of corticosteroid-correlated adverse effects, including gastrointestinal hemorrhage (IRR, 2.62 [1.51-4.54]; IR, 0.02 versus $0.01 ; P<0.001)$, hyperglycemia requiring treatment (IRR, 2.84 [2.47-3.27]; IR, 0.30 versus $0.10 ; P<0.001$ ), infection requiring acceleration of antibiotics (IRR, 4.63 [4.23-5.07]; IR, 0.88 versus $0.19 ; P<0.001$ ), fungal infection needing antifungal treatment (IRR, 3.82 [2.78-5.25]; IR, 0.07 versus $0.02 ; P<0.001)$ and hypernatremia (IRR, 3.09 [2.27-4.20]; IR, 0.06 versus $0.02 ; P<0.001$ ), were all significantly more than the non-corticosteroid group (Table 8). The Cox time-varying analysis indicated that treatment of corticosteroid was associated with significantly increased risks of hyperglycemia and infection with adjusted HR of $3.15(95 \% \mathrm{Cl}, 2.61-3.81 ; P<0.001)$ for hyperglycemia requiring treatment, $2.72(95 \% \mathrm{Cl}, 2.38-3.12 ; P<0.001)$ for infection requiring acceleration for antibiotics. The elevated risks of hyperglycemia requiring treatment (adjusted OR, $2.37 ; 95 \% \mathrm{Cl}, 1.32-$ 4.25; $P=0.004$ ) was consistently observed in corticosteroid group using MSM (Table 8). Therefore, among patients with an NLR $\leq 6.12$, there were no detectable survival benefits, but significantly increased adverse effects, in particular hyperglycemia associated with corticosteroid therapy.

\section{Associations of corticosteroid therapy with outcomes in patients with diabetes}

Given the significantly elevated risk of hyperglycemia by corticosteroid treatment and the high prevalence of pre-existing diabetes in patients with COVID-19, it is clinically important to estimate the influence of corticosteroid use in COVID-19 patients with pre-existing diabetes. This is becoming much more urgent and relevant since patients with diabetes are shown to be at remarkably higher risk of death after SARSCoV-2 infection26, and that risk can be further exacerbated when the blood glucose is poorly controlled27.

In the current study cohort, 2,066 individuals had pre-existing diabetes, among which 596 cases (aged 65.0 [57.0-72.0] years; $59.2 \%$ males) received corticosteroid therapy and the other 1,470 cases (aged 64.0 [57.0-71.0] years; $51.0 \%$ males) did not receive corticosteroids. The occurrences of comorbidities in the corticosteroid and the non-corticosteroid groups in this diabetes cohort were comparable. Patients received corticosteroids exhibited more severe pathological conditions with higher frequencies of lymphocyte decrease and leukocyte count, neutrophil count, CRP, procalcitonin, D-dimer, and organ injury marker increases than those received no corticosteroids.

The incidence of 60-day all-cause death was significantly higher in the diabetic patients taking corticosteroids (IRR, 3.21 [2.47-4.18]; IR, 0.42 versus $0.13 ; P<0.001$ ) compared to those not taking corticosteroids (Table 9). When we addressed the time-varying confounders and imbalanced variables between the corticosteroid and non-corticosteroid groups, there were no significant associations of corticosteroid use with the risk of COVID-19 death in both Cox time-varying model and MSM model (Table 9). 
Regarding the adverse effects, the crude incidences of hyperglycemia requiring treatment (IRR, 2.13 [1.862.43], $P<0.001$ ), infection requiring acceleration of antibiotics (IRR, 4.33 [3.63-5.18], $P<0.001$ ), fungal infection (IRR, 3.45 [2.28-5.23], $P<0.001$ ), and hypernatremia (IRR, 2.40 [1.70- 3.39], $P<0.001$ ) were significantly higher in the corticosteroid group than the non-corticosteroid group (Table 9). After adjusting for time-varying and basic imbalanced confounders, the Cox time- varying and MSM models consistently demonstrated a close association between corticosteroid use and significantly increased risks of hyperglycemia requiring treatment, infection requiring acceleration of antibiotics, needs for antifungal treatment and hypernatremia (Table 9). These findings indicated that, for patients with diabetes, corticosteroid use have more significant impacts on blood glycemic levels and concurrent infections in patients with diabetes compared to the general patients. Despite these side effects, our study did not demonstrate an association between use of corticosteroid and increase or reduction of 60-day all-cause death of COVID-19.

\section{Discussion}

While corticosteroid treatment is viewed as breakthrough progress for COVID-19 therapy28, the specific guideline for the use of corticosteroid in COVID-19 patients is not clearly defined. Giving the major concerns about the variable outcome and the well-demonstrated adverse effects in patients, there is a critical need to develop a highly accurate and robust clinical indicator(s) that will allow optimal patient stratification for maximizing the benefits while minimizing the risk associated with corticosteroid therapy. Here, we performed a retrospective analysis on one of the largest cohorts of hospitalized COVID-19 cases so far reported with more than 12,000 subjects. From the routinely tested blood cells at admission, we identified neutrophil to lymphocyte ratio (NLR) served not only as a highly effective predictor to 60-day all-cause death but also an outstanding indicator for the therapeutic efficacy of corticosteroid therapy at a cut-off value of $>6.12$. NLR can be readily obtained from routine tests for total blood counts, and an NLR based patient stratification has the potential to be established as a highly applicable and costeffective strategy to optimize corticosteroid therapy for COVID-19.

NLR is a well-established indicator of systemic inflammatory status. The NLR dependent association between corticosteroid use and decreased risk of 60-day all-cause death in COVID-19 patients leads more support to the hypothesis that the clinical benefits of in-hospital corticosteroid use were contributed by their favorable immune modulation. For patients with less severe immune response (NLR $\leq 6.12$ ), corticosteroid therapy-associated side effects, in particular hyperglycemia, may offset any potential benefits, leading to no improvements on death rates for COVID-19 patients. Importantly, in the subgroup of patients with diabetes, despite an increased risk of hyperglycemia, use of corticosteroids was not significantly associated with increased mortality. These findings provide valuable insights for the development and implementation of future prospective studies and randomized controlled clinical trials in order to optimize the clinical outcome from corticosteroids in appropriately stratified patients with SARS-CoV-2 infection. 
The results from our study and the RECOVERY trial showed corticosteroid treatment might not bring benefit to all COVID-19 patients11,12. In the RECOVERY trial, patients with ventilation or receiving oxygen showed remarkable reduction in all-cause death by corticosteroid treatment. However, ventilation administration cannot serve as an effective indicator for patient stratification as it can be heavily influenced by many factors, including the availability of medical resources and the clinical experience of the physicians. Our findings revealed that COVID-19 patients with a blood count marker, NLR, above 6.12 taken at admission could see significant protection from corticosteroid therapy. In addition, we validated the accuracy of using NLR 6.12 as a cutoff to predict the favorable outcome of corticosteroids treatment in subgroups with ventilation machine support, in subcategory with methylprednisolone therapy, and patients admitted to different hospitals. Therefore, NLR $>6.12$ is an accurate and robust clinical indicator for predicting beneficial outcome for corticosteroid therapy across different sub-datasets of our COVID-19 cohort.

In addition to sub-cohort differences, the timing, dose, and duration of the corticosteroid treatment could be additional factors contributing to a beneficial or detrimental outcome29. Up to now, evidence on different regimens of corticosteroids has been inconclusive for patients with ARDS30. In our study, among 1,303 patients with NLR $>6.12$ who received corticosteroid treatment, the median daily dosages were $40 \mathrm{mg}$ methylprednisolone-equivalent dose and the initial time was one day after hospital admission, and the median duration was nine days. This indicated that an early intervention with low dosage and short duration of corticosteroids could be helpful for COVID-19 patients with severe inflammatory response. Similar regimen of corticosteroids treatment was also applied in the RECOVERY trial11,12 and another recent trial in patients with ARDS18. In our cohort, methylprednisolone was applied in over $95 \%$ patients 31 ; while dexamethasone, with 5 times more potency than methylprednisolone and longer-lasting effect, was administered in other two trials. As methylprednisolone is more widely used in the treatment of ARDS and septic shock31, it is interesting to investigate if the same NLR parameters and its cut-off value established here are also applicable to different corticosteroid in patient with COVID-19 associated inflammatory morbidities.

It is known for years that severe side effects are associated with corticosteroids treatment and are the sources of great concern in their clinical application. In our study, hyperglycemia was the most common side effect that was associated with corticosteroid therapy in COVID-19 patients. Diabetes is considered a major risk factor for severe outcome in COVID-19 patients, and our previous studies have demonstrated that poorly controlled glycemia was significantly associated with increased risk of death in those individuals27. Therefore, we further explored whether corticosteroids application impacted the clinical outcome in COVID-19 patients with pre-existing diabetes. Our results showed that the use of corticosteroids did not significantly increase 60-day all-cause mortality in COVID-19 patients with diabetes, even when glycemic control was worsened.

While the specific mechanism of action for corticosteroids in COVID-19 therapy remains speculative, it has been well accepted that their use to prevent lung injury under ARDS is largely due to their ability to suppress the devastating systematic inflammation by inhibiting cytokine production and inflammatory 
cell activation. It is now well-recognized that the local lung injury and extrapulmonary organ injury are not only related to a direct virus-induced cytopathic damage, but also a virus infection-triggered dysfunctional immune cell activation, and the ensuing global pro- inflammatory cytokine induction. Indeed, the correlation of NLR with the risk of adverse outcome in COVID-19 as observed in our study further indicate a heightened inflammatory status caused by COVID-19 infection. These mechanistic speculations will need to be further investigated to clarify the molecular and cellular basis for corticosteroid mediated protection in severe cases of COVID-19.

\section{Limitation}

The retrospective design is the main limitation of our study. First, multiple statistical methods were applied to adjust the baseline and time-varying confounders. However, the adjustment may not be adequately balanced bias that could occur in the presence of unmeasured confounding. Second, the cutoff of NLR for indicating the clinical benefits of corticosteroid in the patient subset was mainly calculated and estimated among inpatients in the Chinese population. Whether this cutoff is also applicable for general patients outside of China urgently requires further validations. Third, due to the insufficient laboratory examination data and the relative shortage of medical resources under such an urgent condition of COVID-19 pandemic, we did not assess viral concentration or cleaning rate regarding different treatment groups. Thus, our current study cannot clearly answer whether the virus clearance is impaired by corticosteroid therapy. Fourth, the inherent limitation of an observational research makes it hard to define the causal effects of corticosteroid use on reduced mortality of patients with COVID-19 and with an NLR > 6.12 at admission. Fifth, we did not estimate the risk of patients taking different types of corticosteroids by head-to-head comparisons owing to the limited sample size. Sixth, we did not adjust the variability in the way in which patients have received corticosteroid in enrolled hospitals could add some uncertainty to the results, e.g., patients may initiate corticosteroid earlier than ARDS starts in some hospitals, while, in other hospitals, may use corticosteroid at 24-48 h of ARDS. Further, the difference of dose-dependent associations between corticosteroid administration and COVID-19 outcomes did not evaluate in this study because most patients received a low-to-moderate dosage of methylprednisolone during hospitalization. These limitations highlighted the urgent need for prospective studies and RCTs to further validate the benefits and harms of corticosteroids application in patients according to personalized inflammatory status.

\section{Conclusions}

Our study identified the NLR above 6.12 as a robust and accurate indicator for considering the initiation of corticosteroid treatment in patients with COVID-19. We found that a low dose of corticosteroid treatment in patients with NLR $>6.12$ was associated with a favorably improved 60- day all-cause mortality. In patients with low inflammatory status (NLR $\leq 6.12)$, corticosteroid usage should be used with caution because the significant adverse effects were observed without discernable benefits to 
improve outcomes. Despite the substantial impact on the glycemic control, corticosteroid treatment did not increase 60-day mortality in patients with diabetes. Our study paves the way for designing successful RCTs for investigating the therapeutic effects of corticosteroid therapy in patients suffering from COVID19.

\section{Methods}

\section{Study design and participants}

In this multi-centered, retrospective cohort study, participants diagnosed with COVID-19 and consecutively admitted to the 21 hospitals in Hubei, China that were designated to treat COVID-19 patients were enrolled. A total of 15,649 patients admitted to hospitals from December 30th, 2019 to April 26th, 2020 were enrolled in the study. The final date of follow-up was April 26, 2020. Among these patients, 2,787 patients aged less than 18 , with eGFR $<30 \mathrm{ml} / \mathrm{min} / 1.73 \mathrm{~m} 2$, with liver cirrhosis, with pregnant, with severe medical conditions leading to death, including acute myocardial infarction, acute pulmonary embolism, and stoke, taking corticosteroid for other diseases or less than 3 days, or transferred to other hospitals were excluded.

The throat-swab specimens and/or chest computerized tomography (CT) were examined for all patients upon admission. COVID-19 was diagnosed by clinical manifestations, chest CT, and real- time RT-PCR according to WHO interim guidance and the New Coronavirus Pneumonia Prevention and Control Program (5th edition) published by the National Health Commission of China32,33. The study protocols and procedures were approved by the central ethics committee and were accepted or approved by each collaborating hospital. Ethics committees granted a waiver of the requirement for documentation of informed consent for analyzing existing data without interfering patient treatment.

\section{Data collection and complication evaluation}

We collected patients' demographic information, clinical characteristics, medical history, laboratory tests, radiological reports, therapeutic intervention, and outcome data at admission and during hospitalization at each hospital site. The age, gender, and clinical symptoms (fever, cough, fatigue and dyspnea) were extracted from patients' electronic medical records. Medical histories comprising the coexistence of chronic obstructive pulmonary disease (COPD), type 2 diabetes mellitus (T2DM), hypertension, coronary heart disease, cerebrovascular disease, chronic liver disease, chronic kidney disease, cancer, asthma and autoimmune diseases were reviewed and extracted. The laboratory examination data included a complete blood count, C-reactive protein (CRP), procalcitonin, D-dimer, and serum biochemical test for liver, kidney, heart, and coagulation dysfunction were obtained from the laboratory information system. The unilateral and bilateral lesions in chest CT scan images were analyzed based on the radiological report data. We extracted and analyzed patients' medications and interventions during hospitalization according to doctor advice. Personal identification information (e.g., name and ID) of the study subjects were anonymized and replaced with a coding system before data extraction. We collected data on the daily dose, starting time, duration of each corticosteroid and converted to methylprednisolone-equivalent 
dose. Data were reviewed and confirmed by experienced physicians and were double-checked to guarantee accuracy.

\section{Outcomes and definition}

The primary endpoint was recorded and evaluated in this longitudinal cohort, which was 60-day all- cause death. The gastrointestinal hemorrhage, hyperglycemia requiring treatment, hypernatremia, infection needing acceleration of antibiotics, and fungal infection needing antifungal medication were also recorded and analyzed as adverse effects. The increase of variables was defined as above their upper limits of normal (ULN), and the decrease of variables was defined as below their lower limits of normal (LLN) according to their normal ranges in each hospital site. All primary and secondary endpoints were reviewed and confirmed by a team of certified physicians to ensure accuracy.

\section{Multivariate logistic regression analysis}

The relationship between primary outcome (60-day all-cause mortality) and ten blood cell-related variables among all patients was calculated by the multivariate logistic regression model 26 . The covariates included These blood cell variables comprised white blood cell (WBC) counts increase, neutrophil counts increase, lymphocyte counts decrease, monocyte counts increase, basophil counts increase, eosinophil counts increase, platelet counts decrease, red blood cell (RBC) counts increase, hemoglobin concentrations increase, and hematocrit increase. These predictors were selected based on clinical experience and previous literatures regarding covariates associated with poor outcomes of patients with COVID-19. The odds ratios (OR), 95\% confidential intervals (Cl), and $P$ values were calculated in the multivariate logistic regression analysis.

\section{Cox proportional hazards model accounting for time-varying exposure}

Immortal time bias may arise when patients were waiting for receiving corticosteroid therapy34. When determining clinical outcomes as a time to event, we performed a Cox proportional hazards model accounting for time-varying exposure that adjusted immortal time bias with corticosteroids as a timevarying exposure. When analyzing the association of corticosteroids use with all-cause mortality and adverse effects among patients divided by each cutoff value, we treated corticosteroid initiation as timevarying exposure and adjusted for time-varying exposure and confounders.

\section{Marginal structural model}

Since the time-related changes in patient condition impact the initiation or stop of corticosteroid therapy and thus confound the associations between corticosteroid use and outcomes, we performed marginal structural model (MSM) analysis via the inverse probability of treatment weighting (IPTW) to mitigate time-varying confounders35. CURB-65 pneumonia severity score (confusion, blood urea nitrogen, respiratory rate, SBP, and age)36, neutrophil increase, lymphocyte decrease, and SpO2 levels were timevarying confounders when analyzing the relationship between corticosteroid use with outcomes and 
adverse effects. Imbalanced variables between corticosteroid and non-corticosteroid groups were adjusted.

The stabilized weights for MSM were calculated based on IPTW by multiplying the treatment weights and the censoring weights, where the treatment weights were first derived for each subject to estimate their probability to take corticosteroid therapy at a time, and the censoring weights were calculated to estimate the early time dropout of subjects. The treatment weights were varying until the first day of corticosteroid treatment. The time-varying intercept was assumed as a smooth function and estimated using spline smoothing. The generalized additive model was conducted to estimate the effect of corticosteroids on the results with confounders adjusted. Stabilized weights were pooled into the marginal structural model to calculate the associations between corticosteroid therapy and clinical outcomes.

\section{Sensitivity and subgroup analyses}

For validate NLR 6.12 as an appropriate cutoff for determine using corticosteroids with a favorable outcome, we performed validation using Cox time-varying and MSM models on the following subgroups: (1) patients in the corticosteroid group with NLR $>6.12$ or ::S 6.12 and treated with mechanical ventilation (corticosteroid + ventilation group) versus those on mechanical ventilation but not taking corticosteroids (non-corticosteroid + ventilation group); (2) patients receiving non- methylprednisolone corticosteroid (methylprednisolone group) versus those not taking corticosteroids (non-corticosteroid group); and (3) patients taking corticosteroids versus those not taking corticosteroids after randomly removing two hospitals.

\section{Statistical analysis}

Categorical variables were presented as frequency and continuous variables were described as median (interquartile range, IQR). Means or medians for continuous variables were compared using independent group $t$-tests when the data were normally distributed; otherwise, the Mann-Whitney test was used. Proportions for categorical variables were compared using the $\chi 2$ test. The Fisher exact test was used when the data were limited. The LASSO model was performed using R/glmnet software and the optimal value of $\lambda$ was determined via 10 -fold cross-validations. C-statistic was applied to show the performance of NLR (area under of receiver-operating characteristic curve [AUROC]) in predicting the risk of mortality in the dataset with 12,862 patients with COVID-19. Three cutoff values were selected and their respective accuracy, balanced accuracy, sensitivities, specificities, positive predictive values (PPV), negative predictive values (NPV), and Youden indexes in predicting the risk of death were calculated and compared. We used nonparametric missing value imputation, based on the missForest procedure in the $\mathrm{R} 27,37$, to account for missing data on the laboratory variable. A random forest model using the rest of the variables in the data set was performed to predict the missing values with an estimation of the internally cross-validated errors. The $P$ values were 2 -sided, and an alpha level of 0.05 was used to define statistical significance. Results from all multivariable analyses are reported as odds ratios (OR) or hazard ratios (HR) with $95 \%$ confidence intervals (Cls) as appropriate. All analyses were conducted using $R$ 
version 3.6.3 (R Foundation for Statistical Computing, Vienna, Austria) or SPSS version 23.0 (IBM, Armonk, NY, USA).

\section{Data availability}

The data related to the findings of this study will be available from the corresponding author after publication upon reasonable request. The research team will provide an email address for communication once the data are approved to be shared with others. The proposal with specific aims, statistical plans, and other information/materials may be required to guarantee the rationality of requirements and the security of the data. The patient-level data, but without names and other identifiers, will be shared after review and approval of the submitted proposal and any related requested materials.

\section{Code availability}

The codes used are available from the corresponding authors upon reasonable request.

\section{Declarations}

\section{Acknowledgments}

We thank the patients who participated in this study and thank clinicians who helped to collected and reviewed clinical information. This work was supported by grants from National Key R\&D Program of China (2016YFF0101504, 2019YFC2004700, 2020YFC2004702, 2020YFC0845500), the National Science Foundation of China (81630011, 81970364, 81970070, 81970011, 81870171, 81700356 and

81570412), the Major Research Plan of the National Natural Science Foundation of China (91639304), the Hubei Science and Technology Support Project (2019BFC582, 2018BEC473, and 2017BEC001), and Medical flight plan of Wuhan University.

\section{Author contributions}

JC, Haomiao Li, YML and CZ designed the study, collected and analyzed data, and wrote manuscript. XS, LB, JZ, YCZ, LZ, PZ, ZL, LL, WM, XL, Haofeng Lu, DW, XX, XH, XW, JX, BHZ, and YY collected, reviewed, interpreted, and checked clinical, laboratory, and radiological data. FL, JJQ, and FZ performed statistical analysis. $X Z, J Y, Y X J, G N Z$, edited manuscript and provided valuable suggestions for study design and data analysis. YW, XJZ, ZGS and H.L. contributed equally, designed the project, edited manuscript, and supervised the study. All authors have approved the final version of this paper.

Competing interests. None

\section{References}


1. Barnes, P.J. Kinases as Novel Therapeutic Targets in Asthma and Chronic Obstructive Pulmonary Disease. Pharmacol Rev 68, 788-815 (2016).

2. Cain, D.W. \& Cidlowski, J.A. Immune regulation by glucocorticoids. Nat Rev Immuno/17, 233-247 (2017).

3. Chotiyarnwong, P. \& McCloskey, E.V. Pathogenesis of glucocorticoid-induced osteoporosis and options for treatment. Nat Rev Endocrinol (2020).

4. Russell, C.D., Millar, J.E. \& Baillie, J.K. Clinical evidence does not support corticosteroid treatment for 2019-nCoV lung injury. Lancet (London, England) 395, 473-475 (2020).

5. Tang, C., Wang, Y., Lv, H., Guan, Z. \& Gu, J. Caution against corticosteroid-based COVID- 19 treatment. Lancet (London, England) 395, 1759-1760 (2020).

6. Stockman, J., Bellamy, R. \& Garner, P. SARS: systematic review of treatment effects. PLoS Med 3 , e343 (2006).

7. Arabi, Y.M., et al. Corticosteroid Therapy for Critically III Patients with Middle East Respiratory Syndrome. Am J Respir Crit Care Med 197, 757-767 (2018).

8. Hui, S. Systemic Corticosteroid Therapy May Delay Viral Clearance in Patients with Middle East Respiratory Syndrome Coronavirus Infection. Am J Respir Crit Care Med 197, 700-701 (2018).

9. Ye, Z., et al. Efficacy and safety of corticosteroids in COVID-19 based on evidence for COVID-19, other coronavirus infections, influenza, community-acquired pneumonia and acute respiratory distress syndrome: a systematic review and meta-analysis. CMAJ (2020).

10. Shang, L., Zhao, J., Hu, Y., Du, R. \& Cao, B. On the use of corticosteroids for 2019-nCoV pneumonia. Lancet (London, England) 395, 683-684 (2020).

11. Mahase, Covid-19: Low dose steroid cuts death in ventilated patients by one third, trial finds. $B M J$ (Clinical research ed.) 369, m2422 (2020).

12. Horby, P., et al. Effect of Dexamethasone in Hospitalized Patients with COVID-19 - Preliminary Report. medRxiv (2020).

13. Halpern, D. \& Miller, F.G. The Urge to Build More Intensive Care Unit Beds and Ventilators: Intuitive but Errant. Ann Intern Med (2020).

14. Zhou, F., et al. Clinical course and risk factors for mortality of adult inpatients with COVID- 19 in Wuhan, China: a retrospective cohort study. The Lancet 395, 1054-1062 (2020).

15. Xiong, C., Jiang, L., Chen, Y. \& Jiang, Q. Evolution and variation of 2019-novel bioRxiv (2020).

16. Terpos, E., et al. Hematological findings and complications of COVID-19. American journal of hematology (2020).

17. Rochwerg, B., et al. Corticosteroids in Sepsis: An Updated Systematic Review and Meta- Analysis. Crit Care Med 46, 1411-1420 (2018).

18. Villar, J., et al. Dexamethasone treatment for the acute respiratory distress syndrome: a multicentre, randomised controlled trial. Lancet Respir Med 8, 267-276 (2020). 
19. Siemieniuk, R.A., et al. Corticosteroid Therapy for Patients Hospitalized With Community- Acquired Pneumonia: A Systematic Review and Meta-analysis. Ann Intern Med 163, 519-528 (2015).

20. Wilk, A.J., et al. A single-cell atlas of the peripheral immune response in patients with severe COVID19. Nat Med (2020).

21. Zhang, , et al. Viral and host factors related to the clinical outcome of COVID-19. Nature (2020).

22. Long, , et al. Short-term outcomes of COVID-19 and risk factors for progression. Eur Respir J 55(2020).

23. Liu, Y., et al. Neutrophil-to-lymphocyte ratio as an independent risk factor for mortality in hospitalized patients with COVID-19. J Infect 81, e6-e12 (2020).

24. Qin, , et al. Dysregulation of immune response in patients with COVID-19 in Wuhan, China. Clin Infect Dis (2020).

25. Annane, D., et al. Corticosteroids in the treatment of severe sepsis and septic shock in adults: a systematic review. Jama 301, 2362-2375 (2009).

26. Zhou, F., et al. Clinical course and risk factors for mortality of adult inpatients with COVID- 19 in Wuhan, China: a retrospective cohort study. Lancet (London, England) 395, 1054-1062 (2020).

27. Zhu, L., et al. Association of Blood Glucose Control and Outcomes in Patients with COVID- 19 and Pre-existing Type 2 Diabetes. Cell Metab 31, 1068-1077 e1063 (2020).

28. Ledford, Coronavirus breakthrough: dexamethasone is first drug shown to save lives. in Nature, Vol. 582 (Nature, 2020).

29. Yang, J.-W., Yang, L., Luo, R.-G. \& Xu, J.-F. Corticosteroid Administration for Viral Pneumonia: COVID19 and Beyond. Clin Microbiol Infect (2020).

30. Fadel, R., et al. Early Short Course Corticosteroids in Hospitalized Patients with COVID-19. Clin Infect Dis (2020).

31. Chang, Y.-L., Liu, T. \& Zhu, B. Analysis and comparison of the use of glucocorticoids in 84 hospitals of six cities in China during 2010-2014. Linchuang Yaowu Zhiliao Zazhi 15, 56-61 (2017).

32. National Health Commission of China. New Coronavirus Pneumonia Prevention and Control Program. (National Health Commission of China, http://www.nhc.gov.cn, 2020).

33. World Health Organization. Laboratory testing for 2019 novel coronavirus (2019-nCoV) in suspected human cases Interim guidance. (World Health Organization 2020).

34. Sin, D.D., Man, S.F. \& Tu, J.V. Inhaled glucocorticoids in COPD: immortal time bias. Am J Respir Crit Care Med 168, 126-127 (2003).

35. Zhang, X.J., et al. In-Hospital Use of Statins Is Associated with a Reduced Risk of Mortality among Individuals with COVID-19. Cell Metab (2020).

36. Capelastegui, A., et al. Validation of a predictive rule for the management of community- acquired pneumonia. Eur Respir J 27, 151-157 (2006).

37. Waljee, K., et al. Comparison of imputation methods for missing laboratory data in medicine. BMJ Open 3(2013). 


\section{Tables}

Due to technical limitations, tables 1-9 are only available as a download in the supplemental files section.

\section{Supplementary Files}

This is a list of supplementary files associated with this preprint. Click to download.

- Tables.pdf 